\title{
Partitioning of Anti-inflammatory Steroid Drugs into Phosphatidylcholine and Phosphatidylcholine-Cholesterol Small Unilamellar Vesicles as Studied by Second-Derivative Spectrophotometry
}

\author{
Shigehiko Takegami, ${ }^{*}$ Keisuke Kitamura, Takako Funakoshi, and Tatsuya Kitade \\ Kyoto Pharmaceutical University; 5 Nakauchicho, Misasagi, Yamashina-ku, Kyoto 607-8414, Japan. \\ Received December 5, 2007; accepted February 8, 2008; published online February 14, 2008
}

The partition coefficients $\left(K_{\mathrm{p}} s\right)$ of six anti-inflammatory steroid drugs, dexamethasone (DMS), betamethasone (BMS), triamcinolone acetonide (TCLA), fluocinolone acetonide (FCLA), betamethasone 17,21-dipropionate (BMSDP), and clobetasole propionate (CBSP), for phosphatidylcholine (PC), and PC-cholesterol small unilamellar vesicles (SUVs) were determined by a second-derivative spectrophotometric method. The $K_{p}$ values were obtained with a relative standard deviation of below $10 \%$ and the following order was observed: BMS $\leq$ DMS $<$ TCLA $<$ FCLA $\ll$ BMSDP $<$ CBSP. BMSDP which has a structure that the two hydroxyl groups of BMS are esterified with propionic acid showed a largely enhanced $K_{p}$ value of 10.5 times that of BMS. Further, replacement of a propionate group in BMSDP with a chlorine atom resulted in the highest $K_{\mathrm{p}}$ value (CBSP) within the drugs examined, i.e., the $K_{\mathrm{p}}$ value of CBSP was 1.2 times that of BMSDP. The presence of $30 \mathrm{~mol} \%$ cholesterol in the SUV bilayers reduced these $K_{\mathrm{p}}$ values to approximately $35-50 \%$ of those values for the PC SUVs, although the order of the $K_{\mathrm{p}}$ values remained unchanged. The order of the $K_{\mathrm{p}}$ values agreed with that of the reported dermatological therapeutic potency of these drugs, although the order of their $\log P$ values for $n$-octanol/water systems showed a discrepancy. Our results indicate that the potency of steroid drugs in dermatological treatments depends to some extent on the $K_{\mathrm{p}}$ values of the drug, that is, the affinity of steroid drugs for PC bilayers influences their clinical potency, since potency is related to transdermal absorption. potency

Key words steroid drug; partition coefficient; liposome; second-derivative spectrophotometry; dermatological therapeutic

Synthetic glucocorticoids (anti-inflammatory steroid drugs) are widely used in the treatment of many kinds of inflammatory and allergenic diseases. Following administration, these drugs pass through the cell membrane and exert their action by associating with glucocorticoidal receptors (GRs) in cells. ${ }^{1)}$ The pharmacological action of anti-inflammatory steroid drugs is influenced by ability of GRs to discern between different drug structures. The crucial factor in the therapeutic potency of anti-inflammatory steroid drugs is the binding with the GRs in cells, also, the partitioning of this class of drugs into lipid bilayer membranes plays an important role in potency, as such partitioning affects drug concentrations in the vicinity of GRs.

Partition coefficients of drugs between lipid bilayer vesicles (liposomes) and water provide fundamental information related to drug interactions with biomembranes. In particular, most drugs usually partition into the cell membrane via passive diffusion; such information about drug partitioning has enhanced our understanding of the pharmacodynamics and pharmacokinetics of drugs. Quantitative structure-activity relationship studies of drugs have suggested that the partition coefficients obtained for liposome/water systems are more useful than those obtained for $n$-octanol/water systems. ${ }^{2-4)}$

We have reported that second-derivative spectrophotometry is an important and useful analytical technique that can be applied to determine the partition coefficients $\left(K_{\mathrm{p}} \mathrm{s}\right)$ of certain psychotropic phenothiazine ${ }^{5)}$ and benzodiazepine ${ }^{6}$ drugs between phosphatidylcholine (PC) small unilamellar vesicles (SUVs) and water, without necessitating the troublesome separation procedures ${ }^{7-9)}$ required to eliminate the SUVs, which cause strong light-scattering. Thus, the derivative method has been used in several studies to determine the partition coefficients of drugs between lipid vesicles and water. $^{10-14)}$

In this study, we used second-derivative spectrophotometry to determine the $K_{\mathrm{p}}$ values between PC SUVs and water of the following six anti-inflammatory steroid drugs: dexamethasone (DMS), betamethasone (BMS), triamcinolone acetonide (TCLA), fluocinolone acetonide (FCLA), betamethasone 17,21-dipropionate (BMSDP), and clobetasole propionate (CBSP). In addition to the elimination of background signal effects, an important feature of the derivative method for the determination of the $K_{\mathrm{p}}$ values is that small changes in a spectrum are enhanced. ${ }^{15}{ }^{\mathrm{p}}$ 17) The effects of cholesterol, a major lipid constituent of biomembranes, on the partitioning of these drugs to the PC-cholesterol bilayer of SUVs were also investigated. Furthermore, the relevance of $K_{\mathrm{p}}$ values for the reported therapeutic potency of these six steroid drugs as dermatological treatments is discussed.

\section{Experimental}

Calculation of Molar Partition Coefficients The molar partition coefficient $\left(K_{\mathrm{p}}\right)$ of a steroid drug between SUVs and water is defined as, ${ }^{5,18)}$

$$
K_{\mathrm{p}}=\frac{(\mathrm{mol} \text { of drug in lipid }) /(\mathrm{mol} \text { of lipid })}{(\mathrm{mol} \text { of drug in water }) /(\mathrm{mol} \text { of water })}
$$

This is further described as, ${ }^{5,18)}$

$$
K_{\mathrm{p}}=\frac{\left(\left[\mathrm{S}_{\mathrm{m}}\right] /\left[\mathrm{S}_{\mathrm{t}}\right]\right) /[\mathrm{L}]}{\left(\left[\mathrm{S}_{\mathrm{w}}\right] /\left[\mathrm{S}_{\mathrm{t}}\right]\right) /[\mathrm{W}]}
$$

where $\left[\mathrm{S}_{\mathrm{m}}\right]$ and $\left[\mathrm{S}_{\mathrm{w}}\right]$ represent the concentrations of a steroid drug in SUVs and water, respectively, and $\left[\mathrm{S}_{\mathrm{t}}\right]=\left[\mathrm{S}_{\mathrm{m}}\right]+\left[\mathrm{S}_{\mathrm{w}}\right]$, and $[\mathrm{L}]$ and $[\mathrm{W}]$ are the molar concentrations of lipids ( $\mathrm{PC}+$ cholesterol) in SUV and water $(55.3 \mathrm{M}$ at $37^{\circ} \mathrm{C}$ ), respectively.

If the background signal effect based on SUVs is eliminated in the second derivative spectra, the derivative intensity difference $(\Delta D)$ of a steroid drug before and after the addition of SUVs at a specific wavelength is proportional to the concentration of the steroid drug in the SUVs. As described 
previously, ${ }^{5)}$ we obtain Eq. 2 from Eq. 1 as follows:

$$
\Delta D=\frac{K_{\mathrm{p}} \Delta D_{\max }[\mathrm{L}]}{[\mathrm{W}]+K_{\mathrm{p}}[\mathrm{L}]}
$$

where $\Delta D_{\max }$ is the $\Delta D$ value when all of the steroid drug species in the sample solution are assumed to partition into the SUVs. The values of $K_{\mathrm{p}}$ and $\Delta D_{\max }$ can be calculated from the experimental values of [L] and $\Delta D$ by applying a nonlinear least-squares calculation to Eq. 2. Here, the calculations were performed with a personal computer. ${ }^{5)}$

Reagents DMS, BMS, TCLA, FCLA, BMSDP, and CBSP (Fig. 1) were purchased from Sigma (U.S.A.) and used without further purification. The buffer used in this study was $50 \mathrm{~mm} \mathrm{NaCl}-10 \mathrm{~mm}$ 4-(2-hydroxyethyl)-1piperazineethanesulfonic acid (Hepes buffer, $\mathrm{pH}$ 7.4). L- $\alpha$-PC (egg yolk) of $99 \%$ purity was supplied as a $2 \%(\mathrm{w} / \mathrm{v})$ chloroform solution from Avanti Polar-Lipids Inc. (Alabaster, AL, U.S.A.). Cholesterol (Tokyo Kasei Co., Tokyo, Japan) was recrystallized from ethyl acetate and stored as a $5 \%(\mathrm{w} / \mathrm{v})$ chloroform solution at $-30{ }^{\circ} \mathrm{C}$. The purity of the $\mathrm{PC}$ and cholesterol was confirmed by thin-layer chromatography.

Preparation of PC SUV Appropriate amounts of the PC and cholesterol stock solutions were mixed and dried by using a rotary evaporator and then a vacuum pump. Five milliliters of the buffer was added to the residue in order to yield a PC solution with a concentration of $c a .40 \mathrm{mM} \mathrm{PC}$, and the mixture was vortexed to produce multilamellar vesicles. The SUVs were then prepared by the sonication method, as previously reported. ${ }^{5}$

Measurement of the Mean Diameter of the Vesicles SUV size distribution was determined by a dynamic light-scattering method using a submicron particle analyzer (Nicomp Model 380, Particle Sizing Systems, Santa Barbara, CA, U.S.A.), ${ }^{10)}$ and the diameters of more than $90 \%$ of the SUVs were confirmed to be within a range of $20-30 \mathrm{~nm}^{19}$ )

Phosphorus Determination The exact phospholipid concentrations in the SUV suspensions were calculated from a phosphate analysis conducted according to the phosphovanadomolybdate method. ${ }^{20)}$ The lipid concentrations of the SUV suspensions were given by the sum of the PC and the cholesterol content

Measurements of Absorption and Second Derivative Spectra Sample solutions containing $40 \mu \mathrm{M}$ of one of the following: DMS, BMS, TCLA or FCLA, or $20 \mu \mathrm{M}$ of either BMSDP or CBSP, and various amounts of the SUV suspension were prepared in a manner similar to that described previously. ${ }^{5,10)}$ The reference solutions were prepared without the drugs. Each flask was shaken for a short time and incubated at $37^{\circ} \mathrm{C}$ for $30 \mathrm{~min}$. The absorption spectrum of the sample solution was measured against that of the reference solution by using a spectrophotometer (Hitachi U-3210) equipped with a temperature-regulated cell holder in a 1-cm light-pass length cuvette

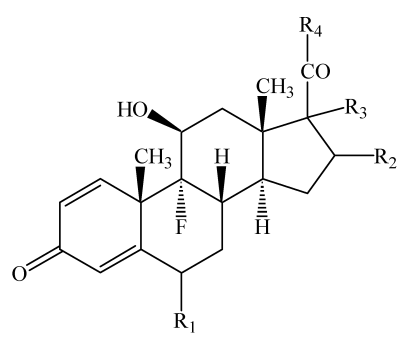

\begin{tabular}{|c|c|c|c|c|}
\hline Drug & $\mathrm{R}_{1}$ & $\mathrm{R}_{2}$ & $\mathrm{R}_{3}$ & $\mathrm{R}_{4}$ \\
\hline DMS & $\mathrm{H}$ & 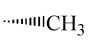 & 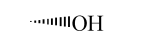 & $\mathrm{CH}_{2} \mathrm{OH}$ \\
\hline BMS & $\mathrm{H}$ & $-\mathrm{CH}_{3}$ & 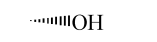 & $\mathrm{CH}_{2} \mathrm{OH}$ \\
\hline TCLA & $\mathrm{H}$ & 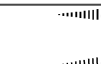 & & $\mathrm{CH}_{2} \mathrm{OH}$ \\
\hline FCLA & …m+1!F & .итाи & $\mathrm{CH}_{3}$ & $\mathrm{CH}_{2} \mathrm{OH}$ \\
\hline BMSDP & $\mathrm{H}$ & $-\mathrm{CH}_{3}$ & $\ldots$ & $\mathrm{CH}_{2} \mathrm{OCOC}_{2} \mathrm{H}_{5}$ \\
\hline CBSP & $\mathrm{H}$ & $-\mathrm{CH}_{3}$ & $\ldots+\cdots+\cdots \mathrm{OCOC}_{2} \mathrm{H}_{5}$ & $\mathrm{CH}_{2} \mathrm{Cl}$ \\
\hline
\end{tabular}

Fig. 1. The Chemical Structures of the 6 Steroid Drugs Studied at $37^{\circ} \mathrm{C}$ and with a band pass of $2 \mathrm{~nm}$, a wavelength interval of $0.1 \mathrm{~nm}$, and a scan speed of $15 \mathrm{~nm} / \mathrm{min}$.

The second derivative spectra based on the Savitzky-Golay method ${ }^{21)}$ were calculated using a personal computer. ${ }^{22)}$ The cubic polynomial convolution of 17 points and a wavelength interval $(\Delta \lambda)$ of $1.0 \mathrm{~nm}$ were used in the calculations.

\section{Results and Discussion}

Absorption and Second Derivative Spectra The absorption spectra of $40 \mu \mathrm{M}$ DMS and FCLA in sample solutions containing various amounts of $\mathrm{PC}$ SUV at $37^{\circ} \mathrm{C}$ are shown in Fig. 2. Both drugs show small spectral changes according to the increases in the PC concentration. Also, no isosbestic point was observed due to the incomplete baseline compensation resulting from the intense light-scattering of the PC SUVs. It is usually difficult to cancel the effects of strong background signals to obtain a flat and zero-level baseline. Thus, further spectral data for calculating the $K_{\mathrm{p}}$ values could not be obtained from these absorption spectra.

The second derivative spectra calculated from the absorption spectra in Fig. 2 are illustrated in Figs. 3a and b, respectively. In these spectra, derivative isosbestic points can be clearly seen at 265 and $283 \mathrm{~nm}$ in the case of DMS, and at $274 \mathrm{~nm}$ for FCLA, thus confirming that the influence of the residual background signal of the PC SUVs was entirely eliminated in the second derivative spectra, and that both drugs were present in two states, ${ }^{23)}$ i.e., in the bulk water and in the PC bilayer of the SUVs. Moreover, the spectral intensity changes of both drugs were clearly enhanced in the second derivative spectra, which enabled us to obtain the exact
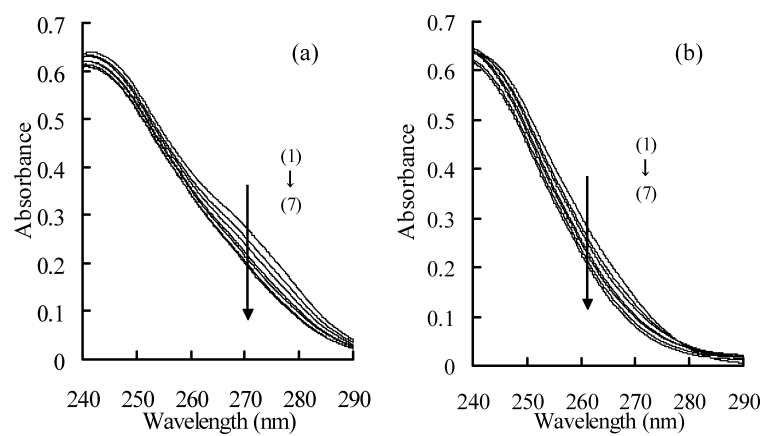

Fig. 2. Absorption Spectra of $40 \mu \mathrm{M}$ DMS (a) and FCLA (b) in Hepes Buffer $\left(\mathrm{pH} 7.4,37^{\circ} \mathrm{C}\right.$ ) Varying Concentrations of PC SUV

PC concentration (mM): (a) (1) 0.0 , (2) 0.4 , (3) 0.8 , (4) 1.2 , (5) 1.6, (6) 2.0, (7) 2.4; (b) (1) 0.0, , (2) 0.4 , (3) 0.8, (4) 1.4, (5) 1.5, , (6) 1.9, , (7) 2.3 (in the direction of the arrow).
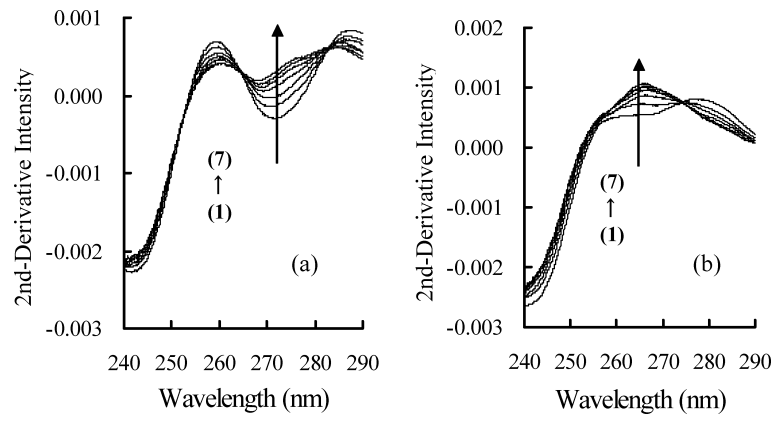

Fig. 3. Second Derivative Spectra of DMS (a) and FCLA (b) Calculated from the Absorption Spectra in Fig. 2

The curve labels are equivalent to those in Fig. 2. 
$\Delta D$ values. Similar results were obtained for BMS, TCLA, BMSDP, and CBSP, and for all of the drugs in the PC-cholesterol SUV experiments.

Calculated $\boldsymbol{K}_{\mathrm{p}}$ Values The $\Delta D$ values used to calculate the $K_{\mathrm{p}}$ and $\Delta D_{\max }$ values were obtained from the derivative values at wavelengths of $273 \mathrm{~nm}$ for DMS, BMS, TCLA, BMSDP, and CBSP, and at a wavelength of $267 \mathrm{~nm}$ for FCLA. Using the obtained $\Delta D$ values, the $K_{\mathrm{p}}$ and $\Delta D_{\max }$ values were calculated by a nonlinear least-squares calculation applied to Eq. 2, and the results are summarized in Table 1. The relative standard deviation (R.S.D.) of each $K_{\mathrm{p}}$ value in Table 1 remained below $10 \%$, thus confirming the precision achieved by the second derivative method.

It has been known that the $K_{\mathrm{p}}$ values of certain amphiphilic drugs show concentration dependence due to the molecular association or formation of their micelles. ${ }^{8,24)}$ To see whether the $K_{\mathrm{p}}$ values of these steroid drugs are affected by their concentrations, the $K_{\mathrm{p}}$ values of DMS and BMS were measured at several concentrations. The results in Table 2 show that the $K_{\mathrm{p}}$ values of each drug were similar for its concentrations employed. The results revealed that neither of these drugs associated nor formed micelles in the bulk water, which in turn confirmed the validity of applying partition theory to account for the interactions between these anti-inflammatory steroid drugs and the PC bilayer.

Lipophilicity-Structure Relationship Based on $K_{\mathrm{p}}$ Values The differences between the $K_{\mathrm{p}}$ values shown in Table 1 indicate the dependence of lipophilicity on molecular structure (see Fig. 1). Thus, using each $K_{\mathrm{p}}$ value as an index for the lipophilicity of the corresponding steroid drug, the effects of chemical structure on lipophilicity can be discussed.

BMS is a stereoisomer of DMS that differs from DMS in terms of the configuration of the C16-methyl group, i.e., BMS has a $\beta$-configuration and DMS an $\alpha$-configuration. Since there is only a small difference between the $K_{\mathrm{p}}$ values of DMS and BMS, it can be concluded that the configuration

Table $1 . K_{\mathrm{p}}$ Values of 6 Steroid Drugs between PC SUV and Water Determined by the Second-Derivative Method

\begin{tabular}{lr}
\hline \hline Drug & $K_{\mathrm{p}}\left(\times 10^{-4}\right)$ \\
\hline DMS & $2.29 \pm 0.20(4)$ \\
BMS & $2.05 \pm 0.05(4)$ \\
TCLA & $2.99 \pm 0.25(3)$ \\
FCLA & $4.85 \pm 0.42(3)$ \\
BMSDP & $21.49 \pm 0.95(3)$ \\
CBSP & $25.62 \pm 1.40(4)$ \\
\hline
\end{tabular}

The mean \pm S.D. Number of the determination is in parentheses. Drug concentration is $40 \mu \mathrm{m}$ for DMS, BMS, TCLA, and FCLA, and $20 \mu \mathrm{m}$ for BMSDP and CBSP, respectively.

Table 2. Dependence of $K_{\mathrm{p}}$ Values of DMS and BMS on Their Concentrations

\begin{tabular}{lcccc}
\hline \hline & \multicolumn{4}{c}{$K_{\mathrm{p}}\left(\times 10^{-4}\right)$} \\
\cline { 2 - 5 } Drug & \multicolumn{4}{c}{ Drug concentration $(\mu \mathrm{M})$} \\
\cline { 2 - 5 } & 20 & 30 & 40 & 60 \\
\hline DMS & $2.19 \pm 0.17(4)$ & $2.36 \pm 0.19(4)$ & $2.29 \pm 0.10(4)$ & $2.28 \pm 0.15(3)$ \\
BMS & $2.11 \pm 0.18(3)$ & $2.07 \pm 0.21(3)$ & $2.05 \pm 0.05(4)$ & $1.99 \pm 0.23(5)$ \\
\hline
\end{tabular}

The mean \pm S.D. Number of the determination is in parentheses. of the C16-methyl group does not affect the partitioning of these steroid drugs into the SUV bilayer.

TCLA and DMS differ structurally in that TCLA has an acetonide group between $\mathrm{C} 16$ and $\mathrm{C} 17$, whereas DMS has methyl (C16) and hydroxyl (C17) groups, respectively. The $K_{\mathrm{p}}$ value of TCLA was found to be approximately 1.3 times higher than that of DMS, showing that the replacement of the methyl and hydroxyl groups by an acetonide group slightly increases the lipophilicity of DMS. Moreover, the $K_{\mathrm{p}}$ value of FCLA (which has a fluorine atom at $\mathrm{C} 6$ with an $\alpha$-configuration, whereas TCLA has a hydrogen atom) increased to approximately 1.6 times that of TCLA, revealing that the substitution of the $\mathrm{H}$ atom with an $\mathrm{F}$ atom considerably increases the affinity for PC bilayers.

Table 1 also shows remarkably large $K_{\mathrm{p}}$ values for BMSDP and CBSP. The structure of BMSDP is such that the two hydroxyl groups at the $\mathrm{C} 17$ and $\mathrm{C} 21$ positions of BMS are esterified by propionic acids. It was confirmed that the esterification of the hydroxyl group with propionic acid greatly strengthened the hydrophobicity of the drug, and resulted in a large increase in the $K_{\mathrm{p}}$ value, i.e., the $K_{\mathrm{p}}$ value of BMSDP increased to approximately 10.5 times that of BMS.

In CBSP, the propionate group at the $\mathrm{C} 21$ position of BMSDP is substituted by a chlorine atom; there was a further increase (1.2 times) in the $K_{\mathrm{p}}$ value of CBSP with respect to that of BMSDP, which renders it the largest among $K_{\mathrm{p}}$ values for these six anti-inflammatory steroid drugs.

Effects of Cholesterol Content on $K_{\mathrm{p}}$ Values Cholesterol is a prominent nonpolar lipid constituent of many biological membranes and it influences interactions between various drugs and biomembranes. Therefore, the effects of cholesterol on the partitioning of these steroid drugs to PCcholesterol bilayers of SUVs were examined. The $K_{\mathrm{p}}$ values for PC-cholesterol SUVs were determined with a range of cholesterol contents of up to $30 \mathrm{~mol} \%$, and are plotted as the ratios to the corresponding $K_{\mathrm{p}}$ values obtained for PC SUVs against the cholesterol content in Fig. 4. The results show that all of the $K_{\mathrm{p}}$ values decreased considerably with increases in cholesterol content, e.g., at a $30 \mathrm{~mol} \%$ cholesterol content, the $K_{\mathrm{p}}$ values decreased to approximately $35-50 \%$ of their values at a $0 \mathrm{~mol} \%$ cholesterol content. It is likely that the decrease in membrane fluidity due to cholesterol ${ }^{25,26}$ ) prevents the interaction between anti-inflammatory steroid drugs and lipid bilayer membranes.

In Fig. 5, the fractions of DMS and CBSP partitioned to

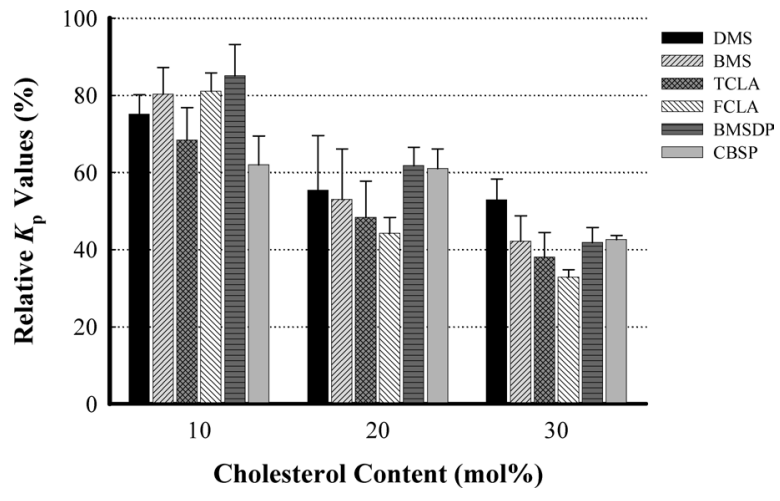

Fig. 4. The Effects of Cholesterol Content in PC-Cholesterol SUV on $K_{\mathrm{p}}$ Values of 6 Steroid Drugs 


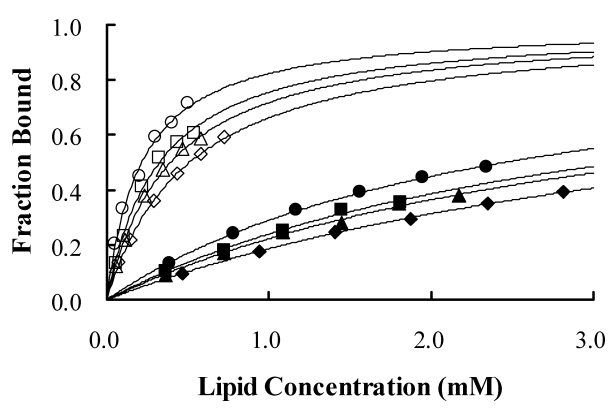

Fig. 5. Fractions $\left(\Delta D / \Delta D_{\max }\right)$ of DMS (Closed) and CBSP (Open) in PCCholesterol SUV Membranes at Various Cholesterol Contents as a Function of Lipid Concentration

The solid lines show the theoretical curves calculated from Eq. 2 using the experimental values of $K_{\mathrm{p}}$ and $\Delta D_{\max }$. The symbols are the experimental values. Cholesterol (mol\%): (circle) 0, (square) 10, (triangle) 20, (diamond) 30.

the PC-cholesterol $(0-30 \mathrm{~mol} \%$ cholesterol $)$ SUVs are shown as a plot of the $\Delta D / \Delta D_{\max }$ values versus lipid concentration. Solid lines represent the theoretical curves calculated from Eq. 2 using the obtained $K_{\mathrm{p}}$ and $\Delta D_{\max }$ values. The experimental values at each cholesterol content show a good fit with the calculated curves, indicating the validity of the obtained $K_{\mathrm{p}}$ values. Similar results were obtained for BMS, TCLA, FCLA, and BMSDP.

Comparison of $K_{p}$ Values with the Dermatological Potency of Steroid Drugs As noted above, the affinity of drugs for biomembranes influences their clinical potency, since potency is related to both the absorption of the drug and its concentration in the membranes of relevant tissues or cells. Therefore, the obtained $K_{\mathrm{p}}$ values were expected to be reflected in the clinical potency of these steroid drugs. Here, we attempted to confirm the relevance of the $K_{\mathrm{p}}$ values to the clinical potency of dermatological treatment, since via the percutaneous absorption, the affinity of drugs for PC bilayers is more directly reflected in the clinical potency of those drugs than via oral or injective administration.

Steroid drugs used in dermatological treatments have been classified into several categories according to their therapeutic potency. ${ }^{27,28)}$ In a recent report, "guideline for therapy for atopic dermatitis 2004", ${ }^{29)}$ several steroid drugs used in topical preparations are classified into 5 categories. In Table 3, the logarithm of the $K_{\mathrm{p}}$ values $\left(\log K_{\mathrm{p}}\right)$ of these steroid drugs and the logarithm of their reported partition coefficients $(\log P)$ determined for $n$-octanol/water systems ${ }^{30)}$ are listed together with this dermatological therapeutic potency (Potency) described in the guideline. ${ }^{29)}$ Herein, BMS is not referred in the guideline. ${ }^{29)}$ However, BMS is widely known to have potency equivalent to that of DMS, ${ }^{31)}$ and therefore, it could be reasonably classified as having medium-level potency (Table 3).

The order of the $\log K_{\mathrm{p}}$ values in Table 3 indeed coincides with that of the dermatological therapeutic potency of these steroid drugs. The results clearly indicate that the potency of steroid drugs as dermatological treatments significantly depends on their $K_{\mathrm{p}}$ values.

On the other hand, the order of the $\log P$ values of the steroid drugs listed in Table 3 does not show complete agreement with that of the dermatological therapeutic potency of these drugs, i.e., the $\log P$ value of CBSP (Strongest), the most therapeutically effective of the drugs listed, is smaller
Table 3. The $\log K_{\mathrm{p}}$ and $\log P$ Values, and Dermatological Therapeutic Potency for the Six Steroid Drugs

\begin{tabular}{llll}
\hline \hline Drug & $\log K_{\mathrm{p}}$ & $\log P^{a)}$ & Potency $^{b)}$ \\
\hline CBSP & 5.41 & 3.83 & Strongest \\
BMSDP & 5.33 & 4.07 & Very strong \\
FCLA & 4.69 & 2.48 & Strong \\
TCLA & 4.48 & 2.30 & Medium \\
DMS & 4.36 & 1.83 & Medium \\
BMS $^{c)}$ & 4.31 & 1.94 & Medium
\end{tabular}

a) Refer to ref. 30 . b) Refer to ref. $29 . \quad$ c) Refer to ref. 31.

than that of BMSDP (Very strong). Thus, it was confirmed that the $K_{\mathrm{p}}$ value of a drug measured in a liposome/water system is a more superior index for evaluating the lipophilicity of a drug than is the $\log P$ value measured in a $n$-octanol/ water system.

In conclusion, it should be emphasized that the $K_{\mathrm{p}}$ values of steroid drugs for the PC liposome/water system were easily and accurately determined using the second-derivative spectrophotometric method. $K_{\mathrm{p}}$ values can therefore serve as indices when assessing lipophilicity-structure relationships, and they are of significant relevance with respect to the therapeutic potency of steroid drugs used as treatments for various dermatological conditions.

Acknowledgements The authors would like to thank Ms. Mayo Fukunaga for her assistance with the experiments.

\section{References}

1) Clark J. H., Schrader W. T., O’Malley B. W., “Textbook of Endocrinology," 8th ed., ed. by Wilson J. D., Foster D. W., Saunders, Philadelphia, 1992, pp. 35-90.

2) Choi Y. W., Rogers J. A., Pharm. Res., 7, 508-512 (1990).

3) Fujiwara H., Da Y. Z., Ito K., Takagi T., Nishioka Y., Bull. Chem. Soc. Jpn., 64, 3707-3712 (1991).

4) Rogers J. A., Choi Y. W., Pharm. Res., 10, 913-917 (1993).

5) Kitamura K., Imayoshi N., Goto T., Shiro H., Mano T., Nakai Y., Anal. Chim. Acta, 304, 101-106 (1995).

6) Omran A. A., Kitamura K., Takegami S., El-Sayed A. Y., Abdel-Mottaleb M., J. Pharm. Biomed. Anal., 25, 319-324 (2001).

7) Bondy B., Remien J., Life Sci., 28, 441-449 (1981).

8) Luxnat M., Galla H. J., Biochim. Biophys. Acta, 856, 274-282 (1986).

9) Zachowski A., Durand P., Biochim. Biophys. Acta, 937, 411-416 (1988).

10) Takegami S., Kitamura K., Kitade T., Hasegawa K., Nishihira A., J. Colloid Interface Sci., 220, 81-87 (1999).

11) Rodrigues C., Gameiro P., Reis S., Lima J. L. F. C., de Castro B., Biophys. Chem., 94, 97-106 (2001).

12) Ferreira H., Lucio M., de Castro B., Gameiro P., Lima J. L. F. C., Reis S., Anal. Bioanal. Chem., 377, 293-298 (2003).

13) Pola A., Michalak K., Burliga A., Motohashi N., Kawase M., Eur. J. Pharm. Sci., 21, 421-427 (2004).

14) Takegami S., Kitamura K., Kitade T., Takashima M., Ito M., Nakagawa E., Sone M., Sumitani R., Yasuda Y., Chem. Pharm. Bull., 53, $147-150$ (2005).

15) Kitamura K., Takenaka M., Yoshida S., Ito M., Nakamura Y., Hozumi K., Anal. Chim. Acta, 242, 131-135 (1991).

16) Kitamura K., Kitade T., Mabuchi M., Mizuochi M., Goto K., Bunseki Kagaku, 42, 581-585 (1993).

17) Hewala I. I., Anal. Lett., 26, 2217-2237 (1993).

18) Welti R., Mullikin L. J., Yoshimura T., Helmkamp G. M., Jr., Biochemistry, 23, 6086-6091 (1984).

19) Kitamura K., Yamamoto M., Takegami S., Sugiura M., Talanta, 49 261-265 (1999).

20) Christopher A. J., Fennell T. R. F. W., Microchem. J., 12, 593-605 (1967).

21) Savitzky A., Golay M. J. E., Anal. Chem., 36, 1627-1639 (1964). 
22) Kitamura K., Hozumi K., Anal. Chim. Acta, 172, 111-118 (1985).

23) Connors K. A., "Binding Constants," John Wiley \& Sons, New York, 1987, pp. 142-147.

24) Charman W. N., Lai C. S., Finnin B. C., Reed B. L., Pharm. Res., 8, 1144-1150 (1991).

25) Demel R. A., Kinsky S. C., Kinsky C. B., Van Deenen L. L. M., Biochim. Biophys. Acta, 150, 655-665 (1968).

26) McElhaney R. N., De Gier J., Van Deenen L. L. M., Biochim. Biophys Acta, 219, 245-247 (1970).

27) Aso M., "Physicians' Therary Manual," Vol. 8, 7(6), Nihon Medis,
Tokyo, 1997.

28) Sato B., "Selecting and Using Glucocorticoids," Nankodo, Tokyo, 2000, pp. 216-219.

29) Furue M., Furukawa F., Hide M., Takehara K., Jpn. J. Dermatol., 114, 135-142 (2004)

30) Hansch C., Leo A., Hoekman D., "Exploring QSAR," American Chemical Society, Washington, DC, 1995.

31) Schimmer B. P., Parker K. L., "Goodman \& Gilman's The Pharmacological Basis of Therapeutics," 11th ed., ed. by Brunton L. L., Lazo J. S., Parker K. L., McGraw-Hill, New York, 2005, p. 1594. 